\title{
Randomized trial of radiotherapy versus radiotherapy plus metronidazole for the treatment metastatic cancer to brain
}

\author{
A Southwest Oncology Group study
}

Harmon J Eyre ${ }^{\text {, J Joel D Ohlsen }}{ }^{\text {I }}$, Jess Frank ${ }^{2}$, Albert F LoBuglio ${ }^{3}$, Joseph D McCracken ${ }^{4}$, TJ Weatherall ${ }^{5} \&$ CM Mansfield 6

${ }^{1}$ University of Utah Medical Center; ${ }^{2}$ Southwest Oncology Group Biostatistical Center; ${ }^{3}$ University of Michigan Medical Center; ${ }^{4}$ Brook Army Medical Center; ${ }^{5}$ Tulane University; ${ }^{6}$ University of Kansas Medical Center

Keywords: brain, metastases, radiotherapy

\section{Summary}

One hundred sixteen eligible patients with metastatic cancer to the brain were randomized to receive either radiotherapy $3000 \mathrm{rad} / 10$ fractions (treatment 1) or the same radiotherapy plus metronidazole $6 \mathrm{gm} / \mathrm{m}^{2}$ (treatment 2). One hundred eleven patients were either fully or partially evaluable. The response rates $(\mathrm{CR}+\mathrm{PR})$ and survival showed no significant differences between treatments. Treatment 1: CR + PR 24\%, median survival 14 weeks, Treatment 2: CR + PR 27\%, median survival 12 weeks. There were no differences observed in response rates based on primary tumor site, neurologic performance status, or extent of metastatic disease. Metronidazole therapy was associated with substantial nausea and vomiting but no neurotoxicity was observed. Oral metronidazole given every other day during radiation therapy provided no clinical benefit for patients with brain metastases compared to radiotherapy alone.

\section{Introduction}

Improvements in cancer therapy have resulted in longer survival in many patients with various forms of malignancy. An unfortunate consequence of this improved survival has been the observation of an increase in metastatic disease to the brain (1-3). Autopsy studies have shown that as many as $18-22 \%$ of patients dying of cancer have intracranial metastases representing over 80000 patients per year in the USA (4-6).

Treatment of metastatic cancer to the brain presents an important palliative problem that has yet to result in substantial survival benefit to the patient (7). Several retrospective studies $(8,9,10)$ and three prospective randomized trials reported by the $\mathrm{Ra}-$ diation Therapy Oncology Group $(11,12)$ have demonstrated the value of whole brain irradiation. There also may be some value in resecting solitary lesions prior to irradiation $(6,13)$.

Even with adequate brain irradiation, $50-60 \%$ of patients with brain metastases still die from this complication $(11,12)$. Thus, more effective treatment is needed. Brain metastases are frequently large masses with areas of necrosis and presumably contain a large fraction of hypoxic cells which are relatively radioresistant. Electron-affinic radiosensitizers, such as metronidazole, have been shown both in vitro and in vivo to enhance radiation sensitivity of hypoxic cells relative to euoxic cells (14). In a randomized trial, radiation plus metronidazole was found to be superior to radiation alone in the treatment of glioblastoma multiforme (15). Based on that experience the Southwest Oncology Group (SWOG) conducted a controlled clinical trial evaluating the effectiveness of metronidazole as a radiosensitizer in the treatment of brain metastases. 


\section{Materials and methods}

Between July 1978 and May 1982, 25 SWOG institutions entered 127 patients on study 7811 . Eligibility requirements included the following: histologic proof of malignancy, clinical documentation of brain metastases by isotope or CT brain scan, neurological symptoms, no prior cranial radiation therapy, expected survival of at least 4 weeks, and informed consent in accordance with institutional policy. All patients were adults. Systemic chemotherapy with agents known to cross the blood brain barrier such as nitrosoureas, procarbazine, DTIC, or high dose methotrexate was not allowed.

Patients were stratified according to the following: site of primary cancer (breast, lung, other); concomitant chemotherapy (yes or no); neurologic status ( $1 \& 2$ vs $3 \& 4$ ); measurable or nonmeasurable intracranial disease and extent of metastases. They were randomly assigned to either radiation therapy alone treatment 1 or radiation therapy plus metronidazole, treatment 2 .

\section{Treatment}

To control for the known palliative benefit of corticosteroids, all patients were given dexamethasone $10 \mathrm{mg} / \mathrm{m}^{2} /$ day in divided doses during the course of radiotherapy. The drug was then tapered and discontinued. If it was necessary to begin steroid therapy again, it was recorded on the data sheets.

\section{Radiation therapy}

All patients were given whole brain radiation through lateral ports encompassing the entire intracranial contents with both ports treated each day. The total dose of 3000 rads calculated at the midplane along the central axis was given in 10 daily fractions using megavoltage equipment or ${ }^{60} \mathrm{Co}$ units.

\section{Metronidazole}

Patients assigned to metronidazole received $6 \mathrm{gm} / \mathrm{m}^{2}$ (maximum dose $10 \mathrm{gm}$ ) in a single dose on treatment days 1, 3, 5, 7 and 9. The drug was given orally 1-4 hours preceding radiation therapy and at least 2-3 hours following the last meal for more dependable absorption. Anti-emetics were given prior to the metronidazole to control nausea and vomiting.

\section{Study parameters and criteria for response}

All patients were followed weekly by history and physical examination to determine neurologic function and performance status. In addition, complete blood counts and chemistry tests including electrolytes, renal function, and liver function were monitored. All patients also had a pre- and post-radiotherapy brain isotope or CT scan. These tests were repeated every 4-8 weeks during follow-up. All patients remained on study until death and an attempt was made to determine if death was due to the brain metastases or systemic disease.

The patients were evaluated for response according to the following criteria:

Complete response: complete disappearance of intracranial disease on isotope or CT brain scan; improvement of neurologic function by at least 1 level and discontinuation of steroids.

Partial response: reduction by $50 \%$ or more of the product of perpendicular diameters of the metastatic disease measured by isotopes or CT brain scan: stable or improved neurologic function and reduced or discontinued steroids.

Stable disease: no significant change in size of brain metastases or change in neurologic function.

Progressive disease: increase in size of brain metastases and deterioration of neurologic function.

\section{Statistical analyses}

Survival curves were estimated by the KaplanMeier technique (16) and compared by the twotailed Gehan's tests (17) and logrank tests (18). Response rates and patient characteristics were compared using ordinary chi square tests. 


\section{Results}

One hundred twenty-seven patients were entered on this study. Of these, 116 were eligible with 111 fully or partially evaluable. The non evaluable cases included 6 with inadequate data submission; 8 who were found unacceptable violations at radiation therapy evaluation; and 2 with failure to receive metronidazole. The patient characteristics of the fully and partially evaluable patients are shown in Table 1. No evidence of statistically significant im-

Table I. Patient characteristics.

\begin{tabular}{lll}
\hline Characteristics & $\begin{array}{l}\text { Radiation } \\
\text { (Treatment } 1)\end{array}$ & $\begin{array}{l}\text { Radiation }+ \\
\text { metronidazole } \\
\text { (Treatment 2) }\end{array}$ \\
\hline Patient no. & 54 & 57 \\
$\quad$ Age $<$ 60 years & 29 & 39 \\
$\quad$ 60 or more years & 25 & 18 \\
Sex M/F & $30 / 24$ & $33 / 24$ \\
Primary site & & \\
Breast & 5 & 8 \\
Lung & 36 & 33 \\
Other & 13 & 16 \\
Neurologic function $1-2$ & 44 & 46 \\
Metastatic extent & 10 & 11 \\
Brain only & & \\
Systemic + brain & 27 & 28 \\
\hline
\end{tabular}

balance in the assignment of patients to treatment was found.

The complete response and partial response rates were similar for both treatments (CR + PR $p$ value $=.958$ ). There was no statistically significant association between the response rates (CR $+\mathrm{PR})$ and the primary site, neurologic function, metastatic extent age or sex. Patients with breast cancer had a higher CR rate but this was not quite statistically significant $(p$-value $=.067)$. These data are listed in Table 2.

Although the median duration of the responses $(\mathrm{CR}+\mathrm{PR})$ was 18 weeks for radiation plus metronidazole compared to 11 weeks for radiation alone, the overall response duration analyses showed no significant difference (Gehan $p$-value $=.442$ ). The four responding patients with breast cancer had a longer duration of response ( 30 weeks median) than those with lung cancer (10 weeks median) (Gehan $p$-values .063). Patients with brain metastases only had no significantly longer response durations than those with both brain and systemic metastases. There were so few patients (5) with poor neurologic function (class $3+4$ ) that responded that the comparison of response duration versus neurologic function is less valid but those with severe impairment had a median response duration of only 7 weeks compared to 18 weeks for neurologic classes

Table 2. Response rate and duration.

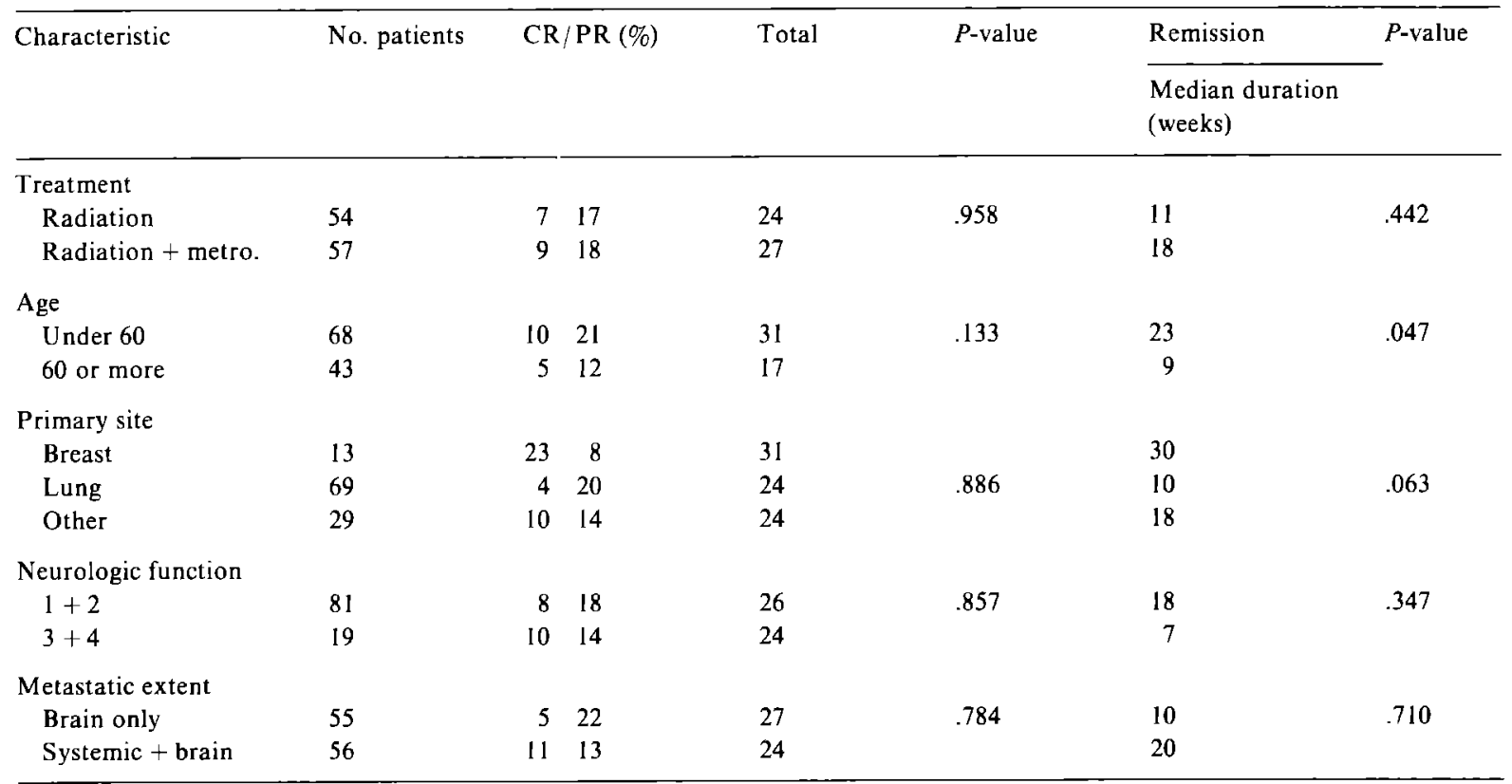


$1+2$. There was a marked difference in remission duration based on age with those under 60 years having longer response durations (Gehan $p$-value $=$ .047). These data are also listed in Table 2 .

The survival of patients treated with radiation alone was not significantly different from those treated with radiation plus metronidazole. These survival curves are illustrated in Fig. 1. Survival based on primary site of malignancy did show a significant difference emerging after about 13 weeks when patients with breast cancer were compared to those with lung cancer (Logrank $p$-value $=.048$ ). This is shown in Fig. 2. Patients with minimal neurological impairment (neurologic class $1+2$ ) had some advantage in survival when compared to those with marked symptoms (neurologic class $3+$ 4) but this difference was not statistically significant (Logrank $p$-value $=.17 \mathrm{I})$ (Fig. 3 ). Regarding the relation of survival duration and extent of metastatic disease no significant difference was observed between patients with only brain metastases and those with brain plus systemic metastases (Gehan $p$-value $=.470)$ as is seen in Fig. 4. Fifty patients $(45 \%)$ were judged by the investigators to have died secondary to their brain metastases. Forty seven $(42 \%)$ died from systemic malignancy and $14(13 \%)$ from both or unknown reasons. Patients aged under 60 years survived significantly longer than those age 60 years or more (Gehan $p$-value $=.037$ ) as shown in Fig. 5.

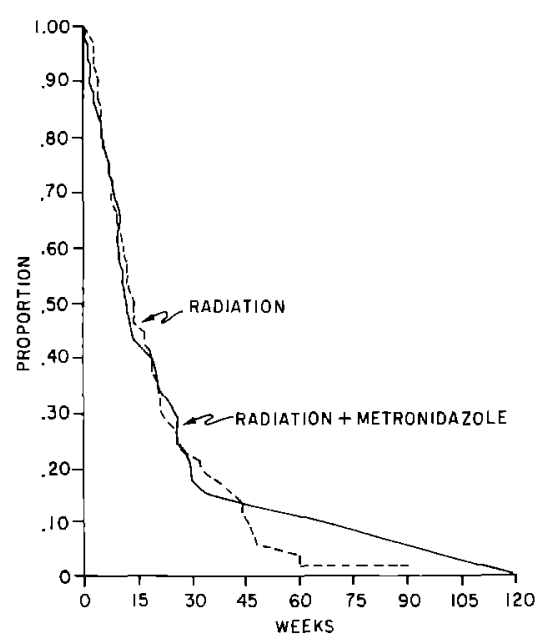

Fig. I. Survival time for all evaluable patients. Treatment 1-radiation only, Treatment 2-radiation plus metronidazole. No significant difference is observed (P-value $=.802)$.

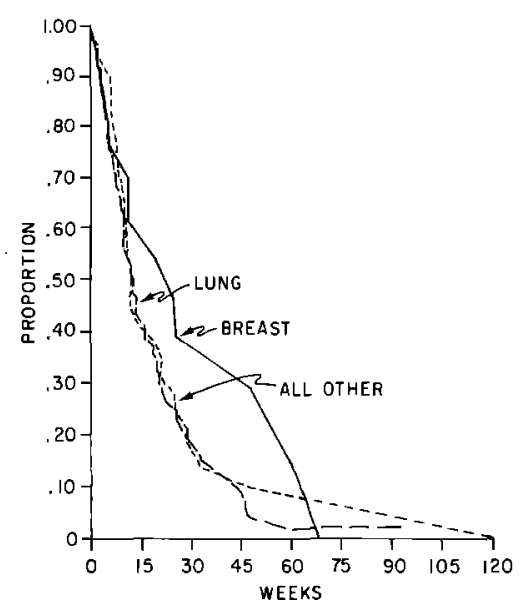

Fig. 2. Survival time based on primary site for all evaluable patients regardless of treatment. There is a significant difference based on the Logrank analysis for breast primary versus lung primary $(P$-value $=.048)$.

All patients developed alopecia and some radiation scalp reaction was noted. These were not significantly worse in patients receiving metronidazole. The major toxicity observed was moderate to severe nausea and vomiting in patients receiving metronidazole. Eighty-two percent of the 57 patients evaluated on treatment 2 were recorded as completing all the prescribed metronidazole. The other ten patients completed 3 or 4 of the 5 doses. The nausea and vomiting was poorly controlled

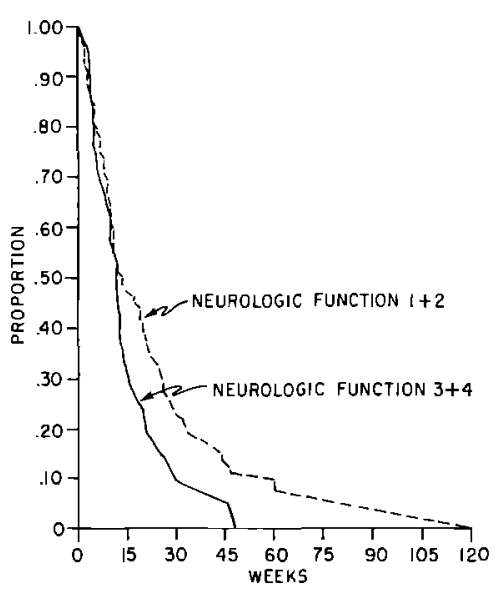

Fig. 3. Survival time for all evaluable patients based on entering neurologic function. No significant difference is observed ( $P$ value $=.171$ ). 


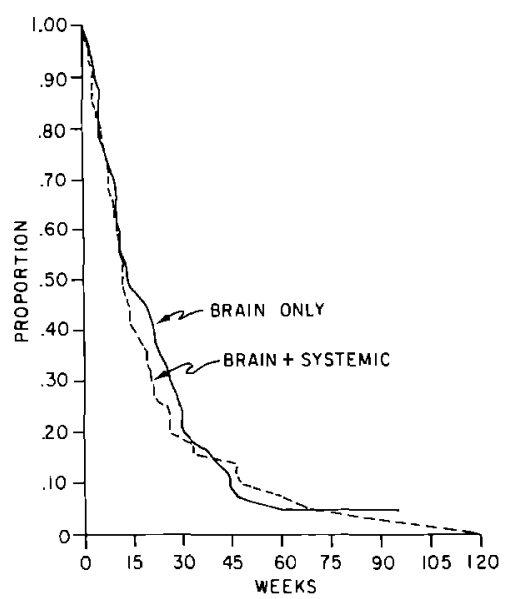

Fig. 4. Survival time for all evaluable patients based on extent of metastases. No significant difference is observed (P-value $=$ $.470)$.

with anti-emetics and clearly is dose limiting with the oral route for this agent. This was limited in time to the day of therapy and did not persist beyond the treatment course. No peripheral neuropathies were seen in the group receiving metronidazole. No other adverse effects were detected. Toxicity data are shown in Table 3.

\section{Discussion}

Metastatic disease to the brain is an ominous occurrence. Almost all patients become sympto-

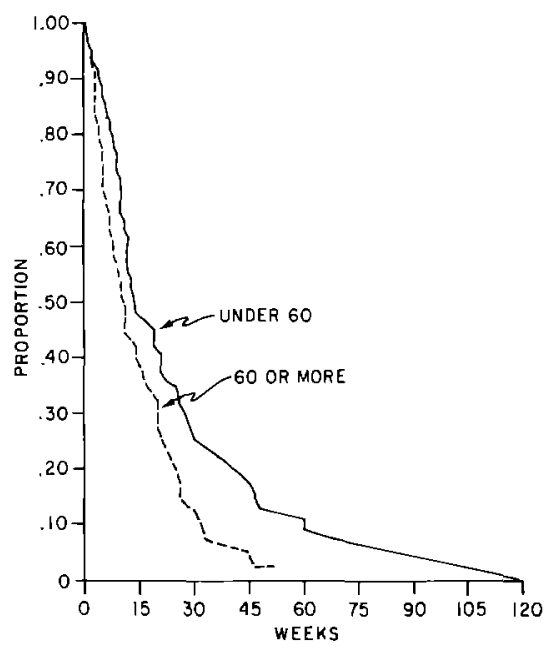

Fig. 5. Survival time for all evaluable patients based on age. Patients less than 60 years survived significantly longer than those 60 years or older $(P$-value $=.037)$.
Table 3. Toxicity.

\begin{tabular}{lrc}
\hline Toxicity & Radiation & $\begin{array}{l}\text { Radiation plus } \\
\text { metronidazole }\end{array}$ \\
\hline Nausea/vomiting & $3.2 \%$ & $51 \%$ \\
Myelosuppression* & $12.9 \%$ & $0 \%$ \\
Neuropathy & $3.2 \%$ & $0 \%$ \\
\hline
\end{tabular}

* Some patients received concomitat chemotherapy with cyclophosphamide, vincristine, and fluorouracil.

matic from it and irregardless of current therapies, the majority will die from this complication within 3-4 months. It is clear that conventional radiotherapy can result in palliative benefit but that it will not result in long term disease control for the majority of patients (7-12). Clearly new therapies are needed.

This randomized clinical trial demonstrates that oral metronidazaole given every other day was not of any value when combined with conventional radiation 300 rad fractions in 10 treatments for treatment of brain metastases compared to radiation alone. Unfortunately other clinical trials using misonidazole have also failed to have significant impact on survival $(19,20)$.

The dose of metronidazole used in this trial resulted in moderate to severe nausea and vomiting which was significant enough to be disabling and dose limiting. If further studies using this agent are undertaken with higher dose some alternate schedule or route of administration will be necessary such as intravenous therapy.

Other ideas under investigation for the treatment of brain metastases such as intra-arterial chemotherapy (21), surgical resection (13), hyperthermia (22), blood brain barrier disruption (23) followed by chemotherapy may be of benefit and should be persued. One other possible approach to this problem would seem to be prophylaxis such as is used with programs designed for small cell lung cancer and leukemia.

\section{Acknowledgements}

This investigation was supported in part by the following PHS grant numbers awarded by the $\mathrm{Na}$ tional Cancer Institute, DHHS: CA-13238, CA12014, CA-27057, CA-22411, CA-03389, CA-12644 and CA-32102. 


\section{References}

1. Espana $P$, Chang $P$, Wiernik PH: Increased incidence of brain metastases in sarcoma patients. Cancer 45:377--380, 1980.

2. Mayer RJ, Berkowitz RS, Griffiths CT: Central nervous system involvement by ovarian carcinoma. A complication of prolonged survival with metastatic disease. Cancer $4 \mathrm{I}$ : 776-783, 1978.

3. Nugent JL, Bunn PA, Jr, Mathews MJ et al.: CNS metastases in small cell bronchiogenic carcinoma. Increasing frequency and changing pattern with lengthening survival. Cancer 44: 1885-1893, 1979

4. Posner JB, Chernik NL: Intracranial metastases from systemic cancer. Adv Neurol 19:579-592, 1978.

5. Aronson SM, Garcia JH, Aronson BE: Metastatic neoplasms of the brain: Their frequency in relation to age. Cancer 17:558-563, 1964.

6. Zimm S, Wampler GL, Stablein D, Hazra T, Young HF: Intracerebral metastases in solid-tumor patients: Natural history and results of treatment. Cancer 48:384-394, 1981.

7. Posner JB: Management of central nervous system metastases. Semin Oncol 4:81-91, 1977.

8. Cairncross $\mathrm{JG}_{7}$ Kim JH, Posner JB: Radiation therapy for brain metastases. Ann Neurol 7:529-541, 1980.

9. Deutsch M, Parsons JA, Mercado R, Jr: Radiotherapy for intracranial metastases. Cancer 34:1607-1661, 1974.

10. Marksbery WR, Books WH, Gupta GD, Young B: Treatment of patients with cerebral metastases. Arch Neurol 35:754-756, 1978.

11. Brogelt B, Gelber R, Kramer S et al.: The palliation of brain metastases: final results of the first two studies by the Radiation Therapy Oncology Group. Int J Radiation Oncology Biol Phys 6:1-9, 1980.

12. Kurtz JM, Gelber R, Brady LW, Carella RJ, Cooper JS: The palliation of brain metastases in a favorable patient population: A randomized clinical trial by the Radiation Therapy
Oncology Group. Int J Radiation Oncology Biol Phys 7:89I-895, 1981 .

13. White KT, Fleming TR, Lows ER: Single metastases to the brain - Surgical treatment in 122 consecutive patients. Mayo Clin Proc 56:424-428, 1981.

14. Chapman JD, Reuvers AP, Borsa J et al.: Nitroheterocytic drugs as selective radiosensitizers of hypoxic mammalian cells. Cancer Chemother Rep 58:559-570, 1974.

15. Urtasun R, Band P, Chapman JD et al.: Radiation and high-dose metronidazole in supratentorial glioblastomas. New Engl J Med 294:1364-1367, 1976.

16. Kaplan EL, Meier P: Nonparametic estimation from incomplete observations. J Am Stat Assoc 53:457-481, 1958.

17. Gehan EA: A generalized Wilcoxin test for comparing arbitrarily singly-censored samples. Biometricha 52:203-223. 1965.

18. Mantel N: Evaluation of survival data and two new rank order statistics arising in its consideration. Cancer Chemotherapy Rep 50:163-170, 1966.

19. Phillips TL, Wasserman TH, Johnson RJ et al.: Final report on the United States phase I clinical trial of the hypoxic cell radiosensitize, misonidazole (Ro-70-0582; NSC \#261037). Cancer 48:1697-1704, 1981 .

20. Ang KK, VanderScheneren E, Notter G et al.: Split course multiple daily fractionated radiotherapy schedule combined with misonidazole for management of grade III and grade IV glioma. A pilot feasability study of the radiotherapy group of EORTC. Int J Radiation Oncology Biol Phys 8:1657-1664, 1982.

21. Madojewicz S, West CR, Park HC et al.: Phase II study-intra-arterial BCNU therapy for metastatic brain tumors. Cancer 47:653-657, 1981.

22. Salcman M, Samaros GM: Hyperthermia for brain tumors: Biophysical rational. Neurosurgery 9:327-335, 1981.

23. Neuwelt EA, Diehl JT, Vu LH et al.: Monitoring of methotrexate delivery in patients with malignant brain tumors after osmotic blood-brain barrier disruption. Ann Intern Med 94:449-454, 1981 . 